Anastasia Drackert (Bochum), Grit Mehlhorn (Leipzig),

Heike Wapenhans (Berlin)

\title{
Medienkompetenz angehender Russischlehrkräfte: Bestandsaufnahme und Entwicklungspotenzial
}

This article is dedicated to the media literacy of future Russian teachers. With the help of an online questionnaire study, filled out by students from three universities in Berlin, Bochum and Leipzig at two survey times - at the beginning of the semester and the semester - it was examined in which areas the students possess good and less good media skills, which skills they were able to acquire during the course of the semester and which they would like to expand in the near future. In addition, the survey provided initial insights into students' attitudes, e.g. regarding the relevance of certain competences of media literacy for their future job as Russian teachers.

Данная статья посвящена медиаграмотности будущих учителей русского языка. В рамках (онлайн)анкетирования, в котором участвовали студенты из трех учебных заведений Берлина, Бохума и Лейпцига и которое было проведено дважды, в начале и конце семестра обучения, было исследовано, в каких областях и насколько хорошо студенты овладели навыками работы с электронными средствами обучения, какие навыки они смогли приобрести в течение семестра и чему еще хотели бы научиться в ближайшем будущем. Кроме того, в ходе опроса было выявлено отношение студентов к различным аспектам медиаграмотности в их будущей работе в качестве учителей русского языка.

Anastasia Drackert und Katrin Bente Karl (Hg.), Didaktik der slawischen Sprachen

Beiträge zum 2. Arbeitskreis in Innsbruck (19.02.-20.02.2018)

(C) 2019 innsbruck university press, ISBN 978-3-903187-80-1, DOI 10.15203/3187-80-1 


\section{Einleitung}

Dass Medienkompetenz in der heutigen, fortwährend digitaler werdenden Welt als Schlüsselqualifikation der Informationsgesellschaft gilt und für einen erfolgreichen Bildungs- und Berufsweg unabdingbar ist, wurde seit einiger Zeit in unterschiedlichen bildungspolitischen Kontexten erfasst: Im Jahre 2006 benannte die Europäische Union die digitale Kompetenz als eine der acht Schlüsselkompetenzen des lebenslangen Lernens (Ferrari 2013). In Deutschland verständigten sich die Bundesländer im Jahre 2016 auf einen verbindlichen Rahmen „Bildung in der digitalen Welt“" und verpflichteten sich, „dafür Sorge zu tragen, dass alle Schülerinnen und Schüler, die zum Schuljahr 2018/2019 in die Grundschule eingeschult werden oder in die Sek I eintreten, bis zum Ende der Pflichtschulzeit die in diesem Rahmen formulierten Kompetenzen erwerben können" (KMK 2016: 18). Der Erwerb der postulierten Kompetenzen durch die Lernenden setzt voraus, dass die Lehrkräfte selbst über die nötigen digitalen Kompetenzen verfügen ${ }^{1}$, was jedoch auch bei der jungen Generation der Lehrenden nicht ohne Weiteres automatisch vorausgesetzt werden kann. So formuliert die „Strategie zur Digitalisierung in der Hochschulbildung“ (SMWK 2018) als Lernziel für die Studierenden u.a. den „Erwerb von notwendigen Kompetenzen für eine digital geprägte Arbeitswelt“" (ebd.: 6).

Neben den fächerübergreifenden Medienkompetenzen, die Lehrpersonen aller Fächer besitzen sollen, ist es wichtig, vor allem aus der Fachperspektive zu wissen, wie Medien eingesetzt werden können, damit das Lernen eines konkreten Unterrichtsfaches unterstützt und gefördert wird (vgl. GFD 2018). Der in den 1980er Jahren in der Sprachlehrforschung etablierte Bereich des Computer Assisted Language Learning (CALL), der sich

\footnotetext{
${ }^{1}$ Die EU hat die DigCompEdu-Matrix zur Selbsteinschätzung für die digitale Kompetenz Lehrender veröffentlicht (Europäische Union 2017).
} 
momentan zu Multimedia Assisted Language Learning (MALL) entwickelt, lieferte zahlreiche Erkenntnisse zum Potenzial moderner Medien für den Erwerb unterschiedlicher Aspekte fremdsprachlicher Kompetenz (vgl. z.B. Beatty 2010; Heim \& Ritter 2012; Rösler 2010 für einen Überblick). Kenntnisse in Bezug auf die Nutzung moderner Medien im Fremdsprachenunterricht gehören zu den fachspezifischen Kompetenzen von Fremdsprachenlehrenden (vgl. KMK 2017: 44-46) und sollten daher auch im Fokus der Russischlehrkräfteausbildung stehen (vgl. Wapenhans 2014: 257).

Die bisherige Forschung im Bereich der Medienkompetenz konzentrierte sich primär auf Kinder, Jugendliche und Erwachsene (vgl. Blömeke 2017). Nach unseren Erkenntnissen wurden bisher kaum Bestandsaufnahmen bezüglich der Medienkompetenzen von Lehramtsstudierenden vorgenommen (vgl. ebd. sowie Middendorff 2002). Zudem wurden bei diesen Untersuchungen lediglich fachunabhängige Kompetenzen von Lehramtsstudierenden erhoben, z.B. ihre Erfahrungen mit Medienerziehung in der Schule. Von dem Hintergrund der defizitären Forschungslage wollten wir herausfinden, welche Voraussetzungen im Bereich der Medienkompetenz angehende Lehramtsstudierende des Russischen mitbringen, um eine evidenzbasierte Entscheidung darüber zu ermöglichen, welche Kompetenzen in den Vordergrund der Ausbildung gerückt werden sollen. Ein weiteres Ziel der Studie war es herauszufinden, was in ganz unterschiedlichen Ausbildungskontexten im Laufe eines Semesters an Zuwachs im Bereich der Medienkompetenzen erreicht werden kann.

\section{Medienkompetenz und ihre Komponenten}

Die aktuellen Definitionen von Medienkompetenz gehen auf Baacke (1997) zurück, der vier verschiedene Dimensionen herausstellte: Medien- 
kunde, -nutzung, -gestaltung und -kritik. Inzwischen erfolgte eine Ausdifferenzierung und Weiterentwicklung des Begriffes und seine Verankerung in den (Rahmen-)Lehrplänen der einzelnen Bundesländer. So heißt es im Berliner Basiscurriculum Medienbildung: „Medienkompetenz bezeichnet die Kenntnisse, Fähigkeiten und Fertigkeiten, die für ein sachgerechtes, selbstbestimmtes, kreatives und verantwortliches Handeln in einer von Medien wesentlich mitbestimmten Welt notwendig sind" (SenBJF \& MBJS 2015: 13). Die Vermittlung von Medienkompetenz im Rahmen des institutionalisierten Fremdsprachenunterrichts sollte Lernende dazu befähigen, „Medien für die Erweiterung ihrer eigenen fremdsprachlichen kommunikativen und interkulturellen kommunikativen Kompetenz zu nutzen" (Grünewald 2017: 245f). Andreas Grünewald (ebd.: 245) formuliert in Konkretisierung der Subkonzepte (computer literacy, visual literacy, print literacy, media content literacy, media grammar literacy, medium literacy) die folgenden Bestandteile von Medienkompetenz für den Fremdsprachenunterricht:

- „technische Aspekte digitaler Medien kennen;

- Medien rezipieren und interaktive Angebote nutzen können;

- visuelle Symbolsysteme dekodieren können;

- Wissen über Mediensysteme und Fähigkeit zur Medienkritik besitzen;

- $\quad$ über Handlungsfähigkeit in Bezug auf Mediengestaltung verfügen;

- reflexiv und kritisch mit Medien umgehen können.“

Die Medienkompetenzen für den Russischunterricht werden im Folgenden exemplarisch am Beispiel von Sachsen aufgezeigt, da die einzelnen Komponenten hier recht ausdifferenziert erscheinen. In den Russischlehrplänen anderer Bundesländer (z.B. SenBJF \& MBJS 2015, MSW 2014) finden sich ähnliche didaktische Grundsätze, fachbezogene Strategien und Lernziele im Bereich der Medienkompetenz. Im Sächsischen Lehrplan Russisch für das Gymnasium (SMK 2011: VIII) taucht Medienkompetenz als eines der zentralen Bildungs- und Erziehungsziele auf, wobei insbesondere 
der Bereich der kritischen Medienkompetenz betont wird: „Sie [die Lernenden, GM] erweitern und vertiefen ihre Kenntnisse über Medien, Mediengestaltungen und Medienwirkungen. Sie lernen, mediengeprägte Probleme zu erfassen, zu analysieren und ihre medienkritischen Reflexionen zu verstärken.“ Der systematische Medieneinsatz soll „das selbstgesteuerte, problemorientierte und kooperative Lernen" fördern (ebd.) und die Lernenden befähigen, „Wissen und Informationen zu gewinnen, einzuordnen und gezielt zu nutzen" (ebd.: 2). Dem Russischlehrplan zufolge ist der Beschäftigung mit Medien im Russischunterricht als zweite Fremdsprache einer von vier (bzw. für Russisch als dritte Fremdsprache einer von drei) Lernbereichen mit Wahlpflichtcharakter gewidmet (vgl. Tab. 1):

\begin{tabular}{|l|l|l|}
\hline Russisch & Klassenstufe & $\begin{array}{l}\text { Lernbereich mit Wahlpflichtcha- } \\
\text { rakter }\end{array}$ \\
\hline $\begin{array}{l}\text { als zweite Fremd- } \\
\text { sprache }\end{array}$ & $6 / 7$ & Mein Computer kann auch Russisch \\
\hline & $8 / 9$ & $\begin{array}{l}\text { Mein Computer kann noch mehr Rus- } \\
\text { sisch }\end{array}$ \\
\hline $\begin{array}{l}\text { als dritte Fremdspra- } \\
\text { che }\end{array}$ & 8 & Russische Massenmedien \\
\hline & 9 & Mein Computer kann auch Russisch \\
\hline & 10 & $\begin{array}{l}\text { Mein Computer kann noch mehr Rus- } \\
\text { sisch }\end{array}$ \\
\hline
\end{tabular}

Tab. 1 Medienkompetenzen im Sächsischen Russischlehrplan (SMK 2011: 6f)

Diesen Lernbereichen werden konkrete Lernziele und -inhalte zugeordnet:

- Beherrschen des russischen Tastaturlayouts,

- Anwenden der aus dem Englischen übernommenen computerbezogenen Lexik (Transliteration der englischen Lexik),

- Gestalten von E-Mails auf Russisch, 
- Einblick gewinnen in die Nutzung russischsprachiger Internetseiten,

- Gestalten einer russischsprachigen Präsentation auf Grundlage einer Internetrecherche (zum Thema ,Russland aktuell“"), z.B. russische Schulhomepage, Popzeitschrift, Tourismusseite, Wandzeitung,

- Einblick gewinnen in russische Massenmedien,

- Auswählen und Nutzen von Medienangeboten, z.B. Printmedien, audio-visuelle Medien, Internet,

- Beschäftigung mit Werbung in den Massenmedien (ebd.: 14-25).

Im Grundkurs und Leistungskurs der Jahrgangsstufen 11/12 kommen weitere Lernziele und -inhalte hinzu, u.a.

- das Ergründen der Rolle der Medien in Deutschland und russischsprachigen Ländern,

- $\quad$ eigenständige und kritische Recherche in Nachschlagewerken, Internet, Sekundärliteratur,

- Verwenden geeigneter Medien, Sprach- und Visualisierungstechniken (richtiges Zitieren, Quellenangabe),

- Kennen der Veränderungen in der russischen Gesellschaft seit der Perestroika,

- Massenmedien und Werbung,

- selbstständige Informationsbeschaffung, Organisation und Auswerten von Informationsmaterial aus verschiedenen Quellen,

- Umgang mit Sekundärliteratur (ebd.: 39-49).

Darüber hinaus stellen Medien einen thematischen Bereich des fächerverbindenden Unterrichts dar (ebd.: XII). So wird im Russischlehrplan auch auf bereits vorhandene Medienkompetenzen aus dem Informatik-, Deutsch- und Englischunterricht verwiesen, an die im Russischunterricht angeknüpft werden soll. 


\section{Studie}

\subsection{Forschungsfragen}

Ziel der Untersuchung war es festzustellen, über welche Kompetenzen angehende Russischlehrende verfügen und inwieweit die Förderung dieser Kompetenzen schon im Laufe eines Semesters an der Hochschule möglich ist.

Für die Studie wurden fünf Forschungsfragen (FF) formuliert:

1. Welche Medien benutzen die Studierenden beim Lernen von Fremdsprachen?

2. In welchen Bereichen der Medienkompetenz verfügen mehr als $70 \%$ der Studierenden über sehr gute Kenntnisse bzw. Erfahrungen?

3. In welchen Bereichen der Medienkompetenz verfügen die Studierenden über sehr wenige bis gar keine Kompetenzen?

4. Welche Kompetenzen möchten sich die Studierenden gern aneignen?

5. Welche Kompetenzen empfinden die Studierenden als irrelevant für ihre künftige Tätigkeit als Russischlehrende?

\subsection{Erhebungsinstrument}

Für die Untersuchung der Forschungsfragen wurde ein Fragebogen eingesetzt. Bei der Suche nach definierten Medienkompetenzen für Lehramts- 
studierende haben wir uns zunächst am Europäischen Portfolio für angehende Fremdsprachenlehrende (EPOSTL, Newby et al. 2007) orientiert. Im Kapitel Resources (ebd.: 30) werden Medienkompetenzen in Anlehnung an den Gemeinsamen europäischen Referenzrahmen für Sprachen (Europarat 2001) in Form von Kann-Beschreibungen formuliert, die der Selbsteinschätzung der Lehramtsstudierenden dienen sollen:

1. „I can identify and evaluate a range of coursebooks/materials appropriate for the age, interests and the language level of the learners.

2. I can select those texts and language activities from coursebooks appropriate for my learners.

3. I can locate and select listening and reading materials appropriate for the needs of my learners form a variety of sources, such as literature, mass media and the Internet.

4. I can make use of ideas and materials included in teachers' handbooks and resource books.

5. I can design learning materials and activities appropriate for my learners.

6. I can recommend dictionaries and other reference books useful for my learners.

7. I can guide learners to produce materials for themselves and for other learners.

8. I can select and use ICT [Information and Communication Technology, GM] materials and activities in the classroom which are appropriate for my learners.

9. I can design ICT materials and activities appropriate for my learners.

10. I can guide learners to use the Internet for information retrieval.

11. I can use and critically assess ICT learning programmes and platforms“" (Newby et al. 2007: 31f). 
Da sich (Medien-)Kompetenzen idealerweise ständig weiterentwickeln, ist es im Sinne des Prozesscharakters von Portfolios, solche Checklisten in verschiedenen Stadien der Lehrerausbildung zu bearbeiten - sowohl um „blinde Flecken“ sichtbar und somit der Reflexion zugänglich zu machen als auch um sich seiner individuellen Kompetenzen bewusst zu werden. Eine Checkliste zur Medienkompetenz kann als Portfolioelement in der Fremdsprachenlehrkräfteausbildung genutzt, für Zwecke der Vergleichbarkeit aber gleichzeitig auch als statistisch auswertbarer Fragebogen konzipiert und zu mehreren Erhebungszeitpunkten eingesetzt werden.

Die im EPOSTL formulierten Kompetenzen sind zwar im Wesentlichen auch heute noch aktuell, sollten jedoch weiter ausdifferenziert und durch neuere Entwicklungen digitaler Medien in den letzten zehn Jahren erweitert werden. Schließlich wurden in unseren Fragebogen solche Kompetenzen aufgenommen, über die angehende Lehrpersonen verfügen sollten, um die in den Lehrplänen verankerten speziellen Kompetenzen für den Russischunterricht bei ihren künftigen Lernenden vermitteln zu können. Die Bereiche „technische Medienkompetenz“, „kritische Medienkompetenz“ sowie „Mediennutzung für den Russischunterricht und die eigene Fortbildung" kristallisierten sich dabei als Schwerpunkte heraus, die nochmals in mehrere Unterkategorien aufgeschlüsselt wurden:

\section{Umgang mit Hard-/Software und webbasierten Diensten zum Spra- chenlernen und -lehren}

- Hardware/PC/Handy

- Software (zum Präsentieren, zur Textverarbeitung, zum Lernen)

- Webbasierte Dienste/Anwendungen

\section{Kritischer Umgang mit Quellen und Informationen}

- Rechtliche Grundlagen

- Prüfen und Bewerten von Quellen und Informationen 
- Vergleich von Informationen aus verschiedenen Quellen

\section{Nutzung von Medien für die eigene Fortbildung, die Unterrichtsvor- bereitung und -durchführung}

- Finden und Adaptieren geeigneter Lernmaterialien und Ressourcen

- Kennen und Nutzen von Tools für die Unterrichtsvorbereitung/Erstellung von Lernmaterialien ${ }^{2}$

Da es sich um eine offene Checkliste handelt, war nach jeder Kategorie noch Platz für weitere Items vorgesehen, damit die Studierenden zusätzliche - ihnen relevant erscheinende - Kompetenzen eintragen konnten. Zudem bestand die Möglichkeit, jedes einzelne Item online zu kommentieren. Vor dem Einsatz des Fragebogens wurde die Meinung von je zwei medienaffinen Russischlehrkräften und Lehramtsstudierenden dazu eingeholt. Ihre Rückmeldungen führten nochmals zu Anpassungen und Ergänzungen in der Checkliste.

Der mit 153 Items sehr umfangreiche Fragebogen wurde in Esurv digitalisiert und den Studierenden in elektronischer Form zur Verfügung gestellt, sodass er sowohl am PC als auch am Smartphone/Tablet bearbeitet werden konnte. Die Datenerhebung erfolgte zu Beginn und Ende des Sommersemesters 2018.

\subsection{Teilnehmende}

An der Studie nahmen 46 Studierende des Russischen mit dem Ziel Lehramt an drei Ausbildungsstandorten teil: Humboldt-Universität zu Berlin,

${ }^{2}$ Der vollständige Fragebogen ist unter https://tinyurl.com/y65wb4de. 
Universität Leipzig und Ruhr-Universität Bochum. Zu Semesterbeginn füllten 38 Studierende den Fragebogen aus, zu Semesterende 35 Studierende, wovon 27 Studierende zu beiden Zeitpunkten an der Befragung teilnahmen.

Die 24 TeilnehmerInnen der Humboldt-Universität setzen sich aus 16 Studierenden im BA-Studiengang Russisch mit Lehramtsoption und 8 Studierenden im Master of Education Russisch zusammen. Die BA-Studierenden besuchten zum Zeitpunkt der Fragebogenerhebung das Seminar „Aspekte des Lehrens und Lernens einer Fremdsprache" im Rahmen des Moduls „Grundlagen der Didaktik des Russischunterrichts“. Da die Lehrveranstaltung in einem Raum mit einem Smartboard stattfand, konnten die Studierenden die verschiedenen Präsentations- und Interaktionsmöglichkeiten eines IWB inkl. der dazugehörigen Dokumentenkamera als Lernende beobachten und selbst ausprobieren. Darüber hinaus thematisierte eine Sitzung Medienkompetenz und Medieneinsatz im Russischunterricht, in der die Studierenden nicht nur den Stand ihrer Medienkompetenz reflektierten, sondern auch ihren Lernbedarf bestimmten. Dem Wunsch, sich intensiver mit dem Smartboard auseinanderzusetzen, konnte in der vorlesungsfreien Zeit mit zwei zusätzlichen Workshops entsprochen werden.

Die Studierenden in der Masterphase konnten ihre Medienkompetenz in der Übung „Texte und Medien“ unter Beweis stellen und weiterentwickeln. So wählten sie nach dem Bearbeiten des Fragebogens die Themen ihrer Präsentationen entsprechend der ermittelten Lernbedürfnisse aus. Vorgestellt wurden mittels Lernen durch Lehren Testaufgaben in Moodle, Vokabeltrainer-Apps, Schneiden und Untertiteln von Videosequenzen, Mahara als E-Portfolio, Online-Umfragetools, Urheber- und Lizenzrecht im Umgang mit Videos und Fotos/Illustrationen.

Von der Universität Leipzig füllten jeweils acht Studierende im Staatsexamensstudiengang Russisch für das Lehramt an Gymnasien und Oberschulen den Fragebogen zu Semesterbeginn und Semesterende aus. Alle acht 
Befragten nahmen in dieser Zeit an dem für das vierte Fachsemester vorgesehenen Modul „Didaktik der slawischen Sprachen 1“ teil, die Hälfte davon absolvierte parallel dazu die schulpraktischen Übungen (,Didaktik der slawischen Sprachen 2") an einem Gymnasium, an dem eine interaktive Tafel im Russischunterricht eingesetzt und mit einem digitalen Unterrichtsmanager zum Russischlehrwerk ,Диалог 1“(Adler et al. 2016) gearbeitet wurde. Im ersten Didaktikmodul wurden - passend $\mathrm{zu}$ den jeweils behandelten Themen - am Rande bestimmte medientechnische „Kniffe“ wie das Installieren einer kyrillischen Schreibschrift, das Einfügen von Betonungszeichen und Zeilenzählern in Texten vorgestellt. Die Studierenden mussten im Rahmen ihres Lehramtsportfolios u.a. einen Tandembogen zum Russischlernen erstellen und sich in diesem Zusammenhang mit der Formatierung von Tabellen auseinandersetzen. Eine Seminarsitzung für die Leipziger Studierenden wurde durch die Didaktikkollegin der Humboldt-Universität zu Berlin gestaltet, die eine praktische Einführung in die Arbeit mit der digitalen Tafel im Russischunterricht gab. Die Teilnehmenden an den schulpraktischen Übungen arbeiteten zudem mit dem Unterrichtsmanager, erstellten Tafelbilder für das Smartboard sowie Powerpoint-Präsentationen und Arbeitsblätter für den Einsatz im schulischen Russischunterricht. Im Lehramtsportfolio zum Modul „Didaktik der slawischen Sprachen 1“ bestand eine Aufgabe der Studierenden darin, ihre individuellen Medienkompetenzen zu reflektieren.

Zum Zeitpunkt der Datenerhebung befanden sich sechs Studierende der Ruhr-Universität Bochum im M.Ed.-Studium und nahmen am dritten fachdidaktischen Seminar teil. Das fachdidaktische Studium am Seminar für Slavistik an der RUB besteht aus vier Seminaren. In der ersten Veranstaltung werden die Grundlagen der Fachdidaktik des Russischen gelegt. Im zweiten Seminar „Grundlagen der Literatur- und Mediendidaktik“ wird das Thema Medien explizit behandelt, wobei der Schwerpunkt der Veranstaltung bis dato auf der Literaturdidaktik lag. Das dritte Seminar bereitet die Studierenden auf das kommende Praxissemester vor, das von einem 
aus fünf Präsenzsitzungen bestehenden vierten didaktischen Seminar begleitet wird.

Der Medieneinsatz stand zwar nicht im Fokus des dritten fachdidaktischen Seminars, wurde jedoch bei einzelnen Themen ,mitbehandelt“. Zum Beispiel sollten sich die Studierenden beim Thema Aufgabenorientierung im Russischunterricht mit dem an der RUB entwickelten Online-Spiel „Zum ersten Mal in Russland“3 vertraut machen, dessen Konzeption anschlieBend im Seminar mit webbasierten Aufgaben, primär zum Lernen von Vokabeln und Grammatik ${ }^{4}$, verglichen und diskutiert wurde. Zudem wurden die Studierenden aufgefordert, die Medienausstattung (Medienschrank, Beamer etc.) für jede Seminarsitzung abwechselnd vorzubereiten, damit sie sich auch mit der technischen Seite vertraut machen können.

Nach der Datenauswertung des ersten Fragebogens (Mitte des Semesters) wurden den Bochumer Studierenden die kumulativ ausgewerteten Daten der Studie präsentiert. Sie machten sich zunächst eigenständig mit den Ergebnissen vertraut und sollten ankreuzen, inwieweit bestimmte Kompetenzen bei ihnen ausbaufähig sind oder nicht (Einzelarbeit). Im zweiten Schritt haben die Studierenden jeweils zu zweit einzelne Kompetenzbereiche besprochen (Partnerarbeit). Im Plenum fand die Klärung der übrigen Fragen statt. Basierend auf den individuell ermittelten Bedürfnissen sollten die Studierenden fünf Bereiche identifizieren, in denen sie sich bis Ende des Semesters eigenständig weiterbilden sollten. In der letzten Veranstaltung des Semesters übernahmen die Studierenden die Präsentation jeweils eines einzelnen Bereichs. Die Präsentation sollte dabei auf das Potenzial des Mediums für das Lernen bestimmter Aspekte der russischen Sprache ausgerichtet werden.

\footnotetext{
${ }^{3} \mathrm{https} / / /$ firsttimeinrussia.wixsite.com/russia

${ }^{4} \mathrm{https} / / /$ learningapps.org/index.php?category $=84 \& \mathrm{~s}=$
} 
Die Studierenden der drei Universitäten, die an der Befragung teilnahmen, befanden sich in unterschiedlichen Stadien ihres Lehramtsstudiums und studierten im Durchschnitt im fünften Semester, wobei auch einige Erstsemester (Quereinsteiger mit anerkannten Studienanteilen aus einem Studium in Russland) und Studierende im 12. Semester teilnahmen. Trotz der Heterogenität der Studienkontexte sollen die Ergebnisse für alle drei Standorte der Russischlehrkräfteausbildung gemeinsam dargestellt werden.

\subsection{Ergebnisse}

Für die erste Foschungsfrage werden die Daten aus der Erhebung am Semesteranfang präsentiert, für die Forschungsfragen 2-5 die Ergebnisse der ersten und zweiten Datenerhebung, damit die Veränderungen im Laufe des Semesters sichtbar werden.

Zuerst wollten wir feststellen, welche Medien die Studierenden beim Lernen von Fremdsprachen selbst benutzen, d.h. welche sie aus der Lernerperspektive kennen (FF1). Insgesamt nannten die Studierenden $128 \mathrm{Me}-$ dien, wovon der größte Teil vier Bereichen zuzuordnen ist (vgl. Tab. 2): Geräte, Printmedien, visuelle Medien und Internet. Dabei fällt auf, dass elektronische Geräte, Internet und Apps insgesamt öfter von den Studierenden genannt werden als Print-Medien. Unbekannte Vokabeln werden von den heutigen Studierenden offensichtlich eher online nachgeschlagen als in gedruckten Wörterbüchern. Interessanterweise nennen nur drei Studierende Moodle oder andere Lernplattformen, obwohl diese in der universitären Sprachausbildung sehr verbreitet sind. Zudem werden bestimmte Medien wie E-Tandem oder Korpora, denen in der Fremdsprachendidaktik großes Potenzial für das Sprachenlernen zugeschrieben wird 
(vgl. Дженкова \& Мозгалина 2010, Guhl 2018), überhaupt nicht erwähnt. Gleichzeitig gaben über 90\% der Befragten an, Skype oder Adobe Connect für den Kontakt zu russischen MuttersprachlerInnen zu nutzen.

\begin{tabular}{|c|c|c|}
\hline $\begin{array}{l}\text { Geräte (25) } \\
\text { PC (5) } \\
\text { Smartphone (5) } \\
\text { Computer (2) } \\
\text { Laptop (2) } \\
\text { I-Pad (2) } \\
\text { Tablet (2) } \\
\text { jeweils 1 Nennung: } \\
\text { Handy, Smartboard, Pro- } \\
\text { jektor, DVD-Player, e- } \\
\text { Book-Reader, Beamer, } \\
\text { Rekorder }\end{array}$ & $\begin{array}{l}\text { Print-Medien (26) } \\
\text { Bücher (11) } \\
\text { Lehrbuch (6) } \\
\text { Zeitschriften (3) } \\
\text { Wörterbücher (2) } \\
\text { jeweils 1 Nennung: } \\
\text { Belletristik, Kursbü- } \\
\text { cher, Zeitungen, } \\
\text { Karteikarten }\end{array}$ & $\begin{array}{l}\text { Visuelle Medien (26) } \\
\text { Filme (11) } \\
\text { YouTube (4) } \\
\text { Videos (3) } \\
\text { Fernsehen (3) } \\
\text { DVD (2) } \\
\text { jeweils 1 Nennung: } \\
\text { Serien, Media Player }\end{array}$ \\
\hline $\begin{array}{l}\text { Internet (17) } \\
\text { Internet (11) } \\
\text { Online-Wörterbücher (4), } \\
\text { z.B. dict.cc (2) } \\
\text { jeweils 1 Nennung: } \\
\text { Online-Lexikon, Online- } \\
\text { Plattformen, Webseiten } \\
\text { wie Quizlet, duolingo }\end{array}$ & $\begin{array}{l}\text { Musik und Ton (11) } \\
\text { Musik (3) } \\
\text { CDs (3) } \\
\text { jeweils } 1 \text { Nennung: } \\
\text { Lieder, Audiobooks, } \\
\text { podcasts, Radiopod- } \\
\text { cast, Audios }\end{array}$ & $\begin{array}{l}\text { Apps (8) } \\
\text { Apps (5) } \\
\text { Mobiltelefon (Übersetzer) } \\
\text { (1) } \\
\text { Apps für Sprachenlernen } \\
\text { (1) } \\
\text { Vokabeltrainerapps (1) }\end{array}$ \\
\hline $\begin{array}{l}\text { EDV (4) } \\
\text { PowerPoint (2) } \\
\text { MS Word (1) } \\
\text { MS Office (1) }\end{array}$ & $\begin{array}{l}\text { E-Learning (3) } \\
\text { Moodle (2) } \\
\text { E-Learning-Kurse } \\
\text { (1) }\end{array}$ & $\begin{array}{l}\text { Sonstiges (8) } \\
\text { digitale Medien (2) } \\
\text { soziale Medien (1) } \\
\text { Vokabeltrainer (1) } \\
\text { Literatur (1) } \\
\text { Tafelbild (1) } \\
\text { OH-Folie (1) } \\
\text { Spiele (1) }\end{array}$ \\
\hline
\end{tabular}

Tab. 2 Von den Studierenden genannte Medien beim Lernen von Fremdsprachen $(N=128)$ 
Aus den Antworten der Studierenden lässt sich nicht immer ableiten, wofür bestimmte Medien bzw. Programme (Internet oder MS Office) verwendet wurden. So kann Word z.B. nicht nur für das Verfassen von Texten auf Russisch benutzt werden, sondern auch zur Korrektur der Rechtschreibung.

Zunächst präsentieren wir die Bereiche, in denen die Studierenden ihrer Einschätzung nach über sehr gute Kompetenzen verfügen (FF2), d.h., in denen mehr als 70\% der Befragten entweder ,trifft zu“ oder ,trifft völlig

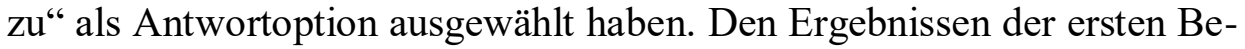
fragung zufolge handelt es sich dabei um 32 Kompetenzen (vgl. Tab. 3), u.a. um das Schreiben auf Russisch und Wechseln zwischen den Sprachen am PC (PowerPoint oder E-Mail) oder auf dem Smartphone (z.B. in WhatsApp), das Erstellen und Bearbeiten von Word-Dateien (Umbenennen, Formatieren, Konvertieren und Scannen), die Nutzung von CD- und DVD-Playern, Tablets, von Internettelefon sowie sozialen Netzwerken. Auch das Nachschlagen unbekannter Vokabeln im Internet oder die Recherche konkreter Informationen zu bestimmten Themen oder grammatischen Fragen scheinen den Studierenden keine Schwierigkeiten zu bereiten. 71,4\% der Befragten teilen zudem mit, Teil eines sozialen Netzwerks zu sein, in dem Russisch verwendet wird, was sicher auch mit dem hohen Anteil an russischsprachigen Studierenden zusammenhängt. In Spalte 4 (Tab. 3) wurden die Werte, die einen Zuwachs zwischen den beiden Befragungen von über $10 \%$ belegen, fett markiert. 


\begin{tabular}{|l|c|c|c|}
\hline Kompetenz & Pre & Post & Zuwachs \\
\hline Ich kann in WhatsApp Russisch schreiben. & 100 & 96,9 & $-3,1$ \\
\hline $\begin{array}{l}\text { Ich kann über WhatsApp Bild-, Video- und } \\
\text { Tondateien austauschen. }\end{array}$ & 100 & 96,9 & $-3,1$ \\
\hline Ich kann einen CD-Player /DVD-Player bedienen. & 97,4 & 100 & 2,6 \\
\hline Ich kann Dateien umbenennen. & 97,2 & 100 & 2,8 \\
\hline $\begin{array}{l}\text { Ich kann die Bedeutung und Verwendung } \\
\text { unbekannter Vokabeln im Internet nachschlagen. }\end{array}$ & 97,1 & 100 & 2,9 \\
\hline $\begin{array}{l}\text { Ich kann auf dem Computer zwischen verschiedenen } \\
\text { Sprachen wechseln. }\end{array}$ & 94,4 & 100 & 5,6 \\
\hline $\begin{array}{l}\text { Ich kann Dateien mit sinnvollen Dateinamen } \\
\text { versehen. }\end{array}$ & 94,4 & 94 & $-0,4$ \\
\hline $\begin{array}{l}\text { Ich kann Dateien (z.B. Word, Bilddateien) in pdf- } \\
\text { Dateien umwandeln. }\end{array}$ & 91,7 & 100 & 8,3 \\
\hline $\begin{array}{l}\text { Ich habe bereits Internettelefon (z.B. Skype, Adobe } \\
\text { Connect) zum Kontakt mit russischen } \\
\text { MuttersprachlerInnen genutzt. }\end{array}$ & 91,4 & 93,5 & 2,1 \\
\hline $\begin{array}{l}\text { Ich komme sowohl mit Word (Pages für MAC) als } \\
\text { auch mit Open Office zurecht. }\end{array}$ & 86,1 & 91,1 & 5 \\
\hline $\begin{array}{l}\text { Ich kann Seitenzahlen, Kopf- und Fußzeilen in } \\
\text { Textdokumente einfügen und bearbeiten. }\end{array}$ & 86,1 & 94,1 & 8 \\
\hline Ich kann an einer Online-Umfrage teilnehmen. & 86,1 & 96,9 & $\mathbf{1 0 , 8}$ \\
\hline $\begin{array}{l}\text { Ich kann eine Lehrwerkübung einscannen und mit } \\
\text { der richtigen Quellenangabe versehen. }\end{array}$ & 85,7 & 90,6 & 4,9 \\
\hline $\begin{array}{l}\text { Ich weiß, wie soziale Netzwerke (z.B. Facebook, } \\
\text { Twitter, vKontakte, Odnoklassniki, Instagram, } \\
\text { Snapchat) funktionieren. }\end{array}$ & 85,7 & 90,3 & 4,6 \\
\hline $\begin{array}{l}\text { Ich kann konkrete Informationen (z.B. } \\
\text { Wetterbericht, Fernsehprogramm, Kinderlieder, } \\
\text { Trickfilme) im russischsprachigen Internet finden. }\end{array}$ & 85,7 & 93,7 & 8 \\
\hline $\begin{array}{l}\text { Ich kann die Aussprache /Betonung einer Vokabel } \\
\text { im Internet überprüfen. }\end{array}$ & 85,7 & 96,8 & $\mathbf{1 1 , 1}$ \\
\hline $\begin{array}{l}\text { Ich kann auf dem Computer Sprachunterstützung } \\
\text { installieren, um bspw. kyrillisch schreiben zu } \\
\text { können und zwischen Deutsch und Russisch zu } \\
\text { wechseln. }\end{array}$ & 83,3 & 100 & $\mathbf{1 6 , 7}$ \\
\hline
\end{tabular}

Anastasia Drackert und Katrin Bente Karl (Hg.), Didaktik der slawischen Sprachen Beiträge zum 2. Arbeitskreis in Innsbruck (19.02.-20.02.2018) 


\begin{tabular}{|l|c|c|c|}
\hline Ich kann einen Screenshot anfertigen und speichern. & 83,3 & 88,2 & 4,9 \\
\hline Ich kann mein Handy zum Vokabellernen benutzen. & 83,3 & 91,1 & 7,8 \\
\hline $\begin{array}{l}\text { Ich kann mich selbst /ein Gespräch mit meinem } \\
\text { Handy /einem Diktiergerät aufzeichnen. }\end{array}$ & 83,3 & 94,1 & $\mathbf{1 0 , 8}$ \\
\hline Ich kann mit einem Tablet umgehen. & 81,1 & 94,1 & $\mathbf{1 3}$ \\
\hline Ich kann Textfelder in ein Dokument einfügen. & 80,6 & 85,2 & 4,6 \\
\hline $\begin{array}{l}\text { Ich weiß, wo ich bei Fragen (z.B. grammatischer } \\
\text { Art) nachschlagen kann. }\end{array}$ & 80 & 96,8 & $\mathbf{1 6 , 8}$ \\
\hline $\begin{array}{l}\text { Ich kann für das Sprachenlernen hilfreiche } \\
\text { Programme/Apps auf meinen Computer } \\
\text { herunterladen. }\end{array}$ & 77,8 & 79,4 & 1,6 \\
\hline $\begin{array}{l}\text { Ich kann Quellen (z.B. Wikipedia-Artikel) in } \\
\text { verschiedenen Sprachen miteinander vergleichen. }\end{array}$ & 77,1 & 93,7 & $\mathbf{1 6 , 6}$ \\
\hline $\begin{array}{l}\text { Ich kann per Skype Dateien verschicken, die ich } \\
\text { während der Sitzung mit meinem Tandempartner } \\
\text { besprechen möchte. }\end{array}$ & 77,1 & 81,2 & 4,1 \\
\hline $\begin{array}{l}\text { Ich kann mich an Abstimmungen (Umfragen) auf } \\
\text { der Lernplattform beteiligen. }\end{array}$ & 75 & 79,4 & 4,4 \\
\hline $\begin{array}{l}\text { Ich kann den Nutzen verschiedener Online- } \\
\text { Wörterbücher für das Fremdsprachenlernen } \\
\text { einschätzen. }\end{array}$ & 75 & 79,4 & 4,4 \\
\hline $\begin{array}{l}\text { Ich kann mich auf englischsprachigen Webseiten } \\
\text { orientieren. }\end{array}$ & 74,3 & 81,2 & 6,9 \\
\hline $\begin{array}{l}\text { Ich kann in E-Mails und Präsentationen problemlos } \\
\text { zwischen Deutsch und Russisch wechseln. }\end{array}$ & 72,2 & 88,2 & $\mathbf{1 6}$ \\
\hline Ich kann mich in ein Doodle eintragen. & 71,4 & 72,7 & 1,3 \\
\hline $\begin{array}{l}\text { Ich bin Teil eines sozialen Netzwerks, in dem } \\
\text { Russisch verwendet wird. }\end{array}$ & 71,4 & 70,9 & $-0,5$ \\
\hline
\end{tabular}

Tab. 3 Überblick über sehr gut ausgebildete Kompetenzen bei mehr als 70\% der Studierenden zu Semesterbeginn („Pre“) und Semesterende („Post“), Angaben in Prozent

$\mathrm{Zu}$ Semesterende verfügen mehr als 70\% der Studierenden ihren Angaben zufolge über 66 unterschiedliche Kompetenzen, demzufolge konnten sie während des Semesters in 34 Bereichen Kompetenzen ausbauen (vgl. Tab. 
4). Wie aus der Anordnung in Tabelle 4 ersichtlich ist, liegt der Zuwachs in der Kompetenzentwicklung zwischen 5,3\% und 47,0\%. Zu den neu gelernten Aspekten gehören u.a. die Einschätzung der Authentizität von Quellen sowie der Umgang mit Quellen von Texten und Materialien, z.B. seriöse von weniger seriösen Quellen unterscheiden, diese vergleichen und kritisch gegenüberstellen. Am Ende des Semesters geben auch viel mehr Studierende an, Online-Wörterbücher und Apps zum Sprachenlernen zu kennen. Hier ist ein Zuwachs von 28,9\% bzw. 30,9\% zu verzeichnen. Einige Items lassen sich den technischen bzw. Software-Kompetenzen zuordnen, darunter Lehrwerkübungen einscannen, Dokumente mit Anderen bearbeiten und teilen (z.B. per Google Drive oder Dropbox), YouTube-Videos herunterladen. Die Fähigkeit zur Einschätzung von Texten und Materialien konnte während des Semesters von besonders vielen Studierenden ausgebaut werden. So trauen sich dies 81,3\% der Befragten zu, während es zu Semesterbeginn nur rund ein Drittel der Studierenden war. Den zweithöchsten Zuwachs verzeichnet das Einscannen von Lehrwerkübungen einschl. der Kennzeichnung der Quellenangabe. Zu Semesterbeginn gab etwas über die Hälfte der Studierenden (57,1\%) an, diese Fähigkeit zu besitzen, und am Ende des Semesters waren es fast alle Befragten (96,9\%). Den Unterschied zwischen sinngemäßer Wiedergabe der Inhalte aus Texten, Zitat und Plagiat kennt zum Semesterende ca. ein Drittel der Befragten mehr als zu Semesterbeginn. Insgesamt sind v.a. im Bereich der kritischen Medienkompetenz Zuwächse zu verzeichnen.

\begin{tabular}{|l|c|c|c|}
\hline Kompetenz & Pre & Post & Zuwachs \\
\hline $\begin{array}{l}\text { Ich kann die Authentizität von Texten und Materialien } \\
\text { einschätzen. }\end{array}$ & 34,3 & 81,3 & 47,0 \\
\hline $\begin{array}{l}\text { Ich kann eine Lehrwerkübung einscannen und mit der } \\
\text { richtigen Quellenangabe versehen. }\end{array}$ & 57,1 & 96,9 & 39,7 \\
\hline $\begin{array}{l}\text { Ich kann bei Übernahmen aus fremden Texten } \\
\text { zwischen sinngemäßer Wiedergabe, Zitat und Plagiat } \\
\text { unterscheiden. }\end{array}$ & 51,4 & 84,4 & 32,9 \\
\hline
\end{tabular}

Anastasia Drackert und Katrin Bente Karl (Hg.), Didaktik der slawischen Sprachen

Beiträge zum 2. Arbeitskreis in Innsbruck (19.02.-20.02.2018)

(C) 2019 innsbruck university press, ISBN 978-3-903187-80-1, DOI 10.15203/3187-80-1 


\begin{tabular}{|l|c|c|c|}
\hline $\begin{array}{l}\text { Ich kann gemeinsam mit Anderen Dokumente } \\
\text { bearbeiten und teilen (z.B. mit Google Drive, Google } \\
\text { Docs). }\end{array}$ & 42,9 & 74,2 & 31,3 \\
\hline Ich kann YouTube-Videos herunterladen. & 57,1 & 88,2 & 31,1 \\
\hline $\begin{array}{l}\text { Ich kenne verschiedene (Online-)Wörterbücher und } \\
\text { weiß, welche für meine Ziele am besten geeignet sind. }\end{array}$ & 62,9 & 93,8 & 30,9 \\
\hline $\begin{array}{l}\text { Ich kann bei Zweifeln an der Glaubwürdigkeit einer } \\
\text { Quelle alternative Quellen zur Überprüfung } \\
\text { heranziehen. }\end{array}$ & 54,3 & 84,4 & 30,1 \\
\hline Ich kenne verschiedene Apps zum Sprachenlernen. & 41,7 & 70,6 & 28,9 \\
\hline $\begin{array}{l}\text { Ich kann Informationen zu einer Person /einem } \\
\text { Ereignis aus unterschiedlichen Quellen } \\
\text { zusammentragen und kritisch gegenüberstellen. }\end{array}$ & 65,7 & 93,8 & 28,0 \\
\hline $\begin{array}{l}\text { Ich kann die inhaltliche Korrektheit von Informationen } \\
\text { im Internet überprüfen. }\end{array}$ & 45,7 & 71,9 & 26,2 \\
\hline $\begin{array}{l}\text { Ich kann seriöse von weniger seriösen Quellen im } \\
\text { Internet unterscheiden. }\end{array}$ & 62,9 & 87,5 & 24,6 \\
\hline $\begin{array}{l}\text { Ich kann die Aktualität von Internetquellen } \\
\text { einschätzen. }\end{array}$ & 60,0 & 84,4 & 24,4 \\
\hline $\begin{array}{l}\text { Ich kann die Rechtschreibhilfe für das Russische } \\
\text { nutzen. }\end{array}$ & 61,1 & 82,4 & 21,2 \\
\hline $\begin{array}{l}\text { Ich kann Kommentare anderer Internetnutzer (z.B. auf } \\
\text { Facebook, YouTube usw.) entsprechend einordnen. }\end{array}$ & 62,9 & 83,3 & 20,5 \\
\hline $\begin{array}{l}\text { Ich kann Dateien in einen Dropbox-/Sciebo- o.̈. } \\
\text { Ordner stellen und teilen. }\end{array}$ & 54,3 & 73,5 & 19,2 \\
\hline $\begin{array}{l}\text { Ich kann Apps für mein eigenes Sprachenlernen } \\
\text { nutzen. }\end{array}$ & 58,3 & 76,5 & 18,1 \\
\hline $\begin{array}{l}\text { Ich kann ein Notebook an einen Beamer und } \\
\text { Lautsprecher anschließen und alles einrichten, um ein } \\
\text { Video zu zeigen. }\end{array}$ & 52,6 & 70,6 & 18,0 \\
\hline $\begin{array}{l}\text { Ich kann Texte von Anderen kommentieren und } \\
\text { korrigieren (z.B. im Änderungsmodus). }\end{array}$ & 52,8 & 70,6 & 17,8 \\
\hline Ich kann einen Overheadprojektor bedienen. & 55,3 & 71,4 & 16,2 \\
\hline
\end{tabular}




\begin{tabular}{|l|c|c|c|}
\hline $\begin{array}{l}\text { Ich weiß, wie verwendete Quellen aus dem Internet } \\
\text { gekennzeichnet und zitiert werden sollen. }\end{array}$ & 65,7 & 81,3 & 15,5 \\
\hline Ich kann Dateien auf einer Lernplattform hochladen. & 63,9 & 79,4 & 15,5 \\
\hline $\begin{array}{l}\text { Ich kann ein Forum innerhalb einer Lernplattform } \\
\text { nutzen. }\end{array}$ & 63,9 & 79,4 & 15,5 \\
\hline $\begin{array}{l}\text { Ich kann die Relevanz von Internetquellen für meinen } \\
\text { Spracherwerb einordnen. }\end{array}$ & 62,9 & 78,1 & 15,3 \\
\hline Ich kann Texte mit einem Zeilenzähler versehen. & 55,6 & 70,6 & 15,0 \\
\hline Ich kann interessante Blogs kommentieren. & 60,0 & 75,0 & 15,0 \\
\hline $\begin{array}{l}\text { Ich kann Tabellen (z.B. für Vokabel- oder Chunk- } \\
\text { Listen) erstellen. }\end{array}$ & 69,4 & 82,4 & 12,9 \\
\hline $\begin{array}{l}\text { Ich kann vorhandene Lernmaterialien adaptieren (z.B. } \\
\text { durch Änderung der Aufgabenstellung, Hinzufügung } \\
\text { aktueller Informationen). }\end{array}$ & 60,0 & 71,9 & 11,9 \\
\hline $\begin{array}{l}\text { Ich kann mit meinem Handy erstellte Audio-, Video- } \\
\text { und Textdateien auf einen Computer überspielen und } \\
\text { weiter bearbeiten. }\end{array}$ & 66,7 & 76,5 & 9,8 \\
\hline Ich kann Tabellen in Excel erstellen und bearbeiten. & 66,7 & 76,5 & 9,8 \\
\hline $\begin{array}{l}\text { Ich kann Anderen (KommilitonInnen /SchülerInnen) } \\
\text { Dateien zum Sprachenlernen zur Verfügung stellen. }\end{array}$ & 63,9 & 73,5 & 9,6 \\
\hline $\begin{array}{l}\text { Ich kann Arbeitsblätter auf Russisch erstellen und } \\
\text { ansprechend formatieren. }\end{array}$ & 61,1 & 70,6 & 9,5 \\
\hline $\begin{array}{l}\text { Ich kann mit Zeilen-, Spalten- und Seitenumbrüchen } \\
\text { umgehen. }\end{array}$ & 63,9 & 70,6 & 6,7 \\
\hline $\begin{array}{l}\text { Ich kann vorhandene Lernmaterialien ergänzen (z.B. } \\
\text { durch Hinzufügen passender Bilder, weiterer Items). }\end{array}$ & 68,6 & 75,0 & 6,4 \\
\hline $\begin{array}{l}\text { Ich kann Internettelefon (z.B. Skype) bewusst zum } \\
\text { Sprachenlernen (Hörverstehen, Sprechen), z.B. mit } \\
\text { einem Tandempartner, nutzen. }\end{array}$ & 65,7 & 71,0 & 5,3 \\
\hline
\end{tabular}

Tab. 4 Überblick über den Kompetenzzuwachs im Laufe des Semesters, Angaben in Prozent 
Ein Kompetenzzuwachs fand auch in einigen Bereichen statt, die bereits zu Semesterbeginn über 70\% der Studierenden gut beherrschten (vgl. Tab. 3). Dazu gehört z.B. das Wissen, wo man bei Fragen (z.B. grammatischer Art) nachschlagen kann (Anstieg von $80 \%$ auf 97\%), die Installation von Sprachunterstützung auf dem Computer, um kyrillisch schreiben und zwischen Deutsch und Russisch wechseln zu können (Anstieg von 83\% auf $100 \%$ ) sowie die Fähigkeit, Quellen wie Wikipedia-Artikel in verschiedenen Sprachen miteinander vergleichen zu können (Anstieg von 77\% auf $94 \%)$.

Neben den Aspekten, in denen die Studierenden über sehr gute Kenntnisse verfügen, wurde auch analysiert, in welchen Bereichen der Medienkompetenz die Studierenden wenige bis gar keine Erfahrungen haben (FF3), d.h. Items, in denen mehr als 60\% der Befragten entweder ,habe ich noch nie verwendet“" oder ,trifft eher nicht zu“ als Antwortoption ausgewählt haben. Wie Tab. 5 zu entnehmen ist, wurden zu Semesterbeginn insgesamt 29 solche Kompetenzbereiche bzw. Medien genannt (zwischen 60\% und 91,4\% der Befragten), zum Semesterende waren es nur noch zehn (zwischen $60 \%$ und 91,4\% der Befragten). Die Markierungen in Spalte 4 heben die Kompetenzen hervor, die zwischen den beiden Befragungen offensichtlich eine Entwicklung erfahren haben.

\begin{tabular}{|l|c|c|c|}
\hline Kompetenz & Pre & Post & $\begin{array}{l}\text { Diff } \\
\text { Post- } \\
\text { Pre }\end{array}$ \\
\hline Ich habe bereits ein Webinar für Andere angeboten. & 91,4 & 90,0 & $-1,4$ \\
\hline $\begin{array}{l}\text { Ich kann Karaoke-Dateien zu russischen Liedern } \\
\text { erstellen. }\end{array}$ & 91,4 & 68,7 & $-22,7$ \\
\hline $\begin{array}{l}\text { Ich kann mit einem entsprechenden Tool (z.B. Quizlet, } \\
\text { Goconqr) virtuelle Lernkartensets erstellen. }\end{array}$ & 88,6 & 59,8 & $\mathbf{- 2 8 , 8}$ \\
\hline $\begin{array}{l}\text { Ich habe bereits selbst eine Videokonferenz organisiert } \\
\text { /moderiert. }\end{array}$ & 85,7 & 78,1 & $-7,6$ \\
\hline $\begin{array}{l}\text { Ich kann mit einem entsprechenden Tool (z.B. Wordle) } \\
\text { Wortwolken auf Russisch erstellen. }\end{array}$ & 85,7 & 69,9 & $-15,8$ \\
\hline
\end{tabular}

Anastasia Drackert und Katrin Bente Karl (Hg.), Didaktik der slawischen Sprachen Beiträge zum 2. Arbeitskreis in Innsbruck (19.02.-20.02.2018)

() 2019 innsbruck university press, ISBN 978-3-903187-80-1, DOI 10.15203/3187-80-1 


\begin{tabular}{|c|c|c|c|}
\hline $\begin{array}{l}\text { Ich kann eine Videosequenzanalyse für die Behandlung } \\
\text { eines Films im Unterricht erstellen. }\end{array}$ & 85,7 & 64,5 & $-21,2$ \\
\hline $\begin{array}{l}\text { Ich kann einen Unterrichtsmanager zu einem aktuellen } \\
\text { Lehrwerk (z.В. Диалог) benutzen. }\end{array}$ & 82,9 & 45,2 & $-37,7$ \\
\hline $\begin{array}{l}\text { Ich kann mit einem entsprechenden Tool } \\
\text { Kreuzworträtsel in russischer Sprache erstellen. }\end{array}$ & 82,9 & 64,8 & $-18,0$ \\
\hline Ich kann selbst Podcasts erstellen. & 82,9 & 64,0 & $-18,8$ \\
\hline Ich kann mit einer Dokumentenkamera arbeiten. & 81,1 & 32,3 & $-48,8$ \\
\hline $\begin{array}{l}\text { Ich kann bei Bedarf auch mit anderen Programmen } \\
\text { (z.B. Prowise Presenter) umgehen. }\end{array}$ & 80,6 & 70,6 & $-10,0$ \\
\hline Ich kann ein Online-Portfolio bearbeiten. & 80,0 & 50,0 & $-30,0$ \\
\hline $\begin{array}{l}\text { Ich kann mit einem entsprechenden Tool MindMaps auf } \\
\text { Russisch erstellen. }\end{array}$ & 80,0 & 40,6 & $-39,4$ \\
\hline Ich habe mich schon aktiv in ein Webinar eingebracht. & 77,1 & 66,7 & $-10,5$ \\
\hline Ich kann selbst Lernvideos erstellen. & 77,1 & 49,8 & $-27,4$ \\
\hline $\begin{array}{l}\text { Ich habe schon einmal an einem Webinar } \\
\text { teilgenommen. }\end{array}$ & 74,3 & 56,7 & $-17,6$ \\
\hline $\begin{array}{l}\text { Ich kann selbst Aufgaben zum Sprachenlernen mit } \\
\text { Vorlagen /Modulbausteinen erstellen, z.B. auf } \\
\text { https://learningapps.org oder mit SMART Lab/Activity } \\
\text { Builder. }\end{array}$ & 72,2 & 51,5 & $-20,7$ \\
\hline $\begin{array}{l}\text { Ich kann eine Webseite (z.B. mit Wordpress) erstellen } \\
\text { und bearbeiten. }\end{array}$ & 71,4 & 68,8 & $-2,7$ \\
\hline Ich kann selbst eine Online-Umfrage erstellen. & 71,4 & 39,4 & $-32,0$ \\
\hline $\begin{array}{l}\text { Ich kann mit einem } I W B \text { erstellte Tafelbilder } \\
\text { abspeichern und sie wieder verwenden. }\end{array}$ & 70,3 & 48,5 & $-21,8$ \\
\hline $\begin{array}{l}\text { Ich kann Präsentationen für die Arbeit mit der } \\
\text { interaktiven Tafel (z.B. mit SMART Notebook, } \\
\text { ActivInspire) erstellen. }\end{array}$ & 69,4 & 51,5 & $-17,9$ \\
\hline $\begin{array}{l}\text { Ich kann Links kürzen, um die Fehlereingabe zu } \\
\text { minimieren. }\end{array}$ & 68,6 & 45,2 & $-23,4$ \\
\hline $\begin{array}{l}\text { Ich kenne die Vorgaben aus dem Lehrplan und weiß, } \\
\text { welche Medienkompetenzen von den SchülerInnen im } \\
\text { Russischunterricht entwickelt werden sollen. }\end{array}$ & 65,7 & 9,4 & $-56,3$ \\
\hline
\end{tabular}

Anastasia Drackert und Katrin Bente Karl (Hg.), Didaktik der slawischen Sprachen 


\begin{tabular}{|l|r|r|r|}
\hline $\begin{array}{l}\text { Ich kann eine Sprachaufnahme mit Hilfe des Computers } \\
\text { verschriftlichen (transkribieren). }\end{array}$ & 63,9 & 50,0 & $-13,9$ \\
\hline Ich kann Standbilder zu Videos erstellen. & 62,9 & 43,3 & $-19,5$ \\
\hline Ich kann selbst ein Doodle erstellen. & 60,0 & 45,5 & $-14,5$ \\
\hline Ich kann selbst einen Blog (auf Russisch) erstellen. & 60,0 & 59,4 & $-0,6$ \\
\hline $\begin{array}{l}\text { Ich kann Potenziale und Grenzen von programmiertem } \\
\text { Feedback zu Online-Übungen und -Tests einschätzen. }\end{array}$ & 60,0 & 37,5 & $-22,5$ \\
\hline Ich kann Untertitel zu Videoausschnitten hinzufügen. & 60,0 & 32,3 & $\mathbf{- 2 7 , 7}$ \\
\hline
\end{tabular}

Tab. 5 Überblick über bis dato nicht ausgebildete Kompetenzen (in Prozent)

Ein Teil der großen Lernfortschritte in bestimmten Bereichen lässt sich auf die im Laufe des Semesters in den Didaktikveranstaltungen besprochenen Themen und durchgeführten Aktivitäten zurückführen. So kannten zu Semesterende fast alle Studierenden die Vorgaben aus dem Lehrplan und wussten, welche Medienkompetenzen von den Lernenden im Russischunterricht entwickelt werden sollen, während zu Semesterbeginn 65,7\% über wenig Wissen in diesem Bereich verfügten. $\mathrm{Zu}$ den weiteren Kompetenzen mit beachtlichem Lernzuwachs gehören die Bedienung von SMARTBoards und Dokumentenkamera, die Arbeit mit dem Unterrichtsmanager zu einem aktuellen Lehrwerk, die Erstellung von MindMaps oder virtuelle Lernkartensets, die Bearbeitung eines Online-Portfolios sowie die Erstellung einer Online-Umfrage.

Kompetenzen, die auch zu Semesterende nur wenige Befragte beherrschten, betrafen spezielle Tools wie Quizlet, Coconqr, Wordle, LearningApps, Programme (z.B. zur Erstellung von Podcasts oder Videos) sowie konkrete Geräte wie die Dokumentenkamera oder die interaktive Tafel. Außerdem fehlten mehreren Studierenden Erfahrungen mit Webinaren, Videokonferenzen und Online-Umfragen. ${ }^{5} \mathrm{Zu}$ den Kompetenzen, die sich mehr

\footnotetext{
${ }^{5}$ Unter den in Tabelle 5 zusammengefassten Kompetenzen waren auch solche, bei denen einige Studierende nicht genau wussten, was gemeint ist. Dazu gehörten Formulierungen,
} 
als 55\% der Studierenden in naher Zukunft gern aneignen wollen (FF4), gehören u.a.:

- Karaoke-Dateien zu russischen Liedern erstellen

- mit einem entsprechenden Tool MindMaps auf Russisch erstellen

- eine Videosequenzanalyse für die Behandlung eines Films im Unterricht erstellen

- bei Bedarf auch mit anderen Programmen (z.B. Prowise Presenter) umgehen

- $\quad$ sich mit dem Urheberrecht auskennen

- Lernvideos und Podcasts erstellen

- Plattformen, die lizenzfreie Musik, Fotos, Clip Art anbieten, kennen

- mit einem entsprechenden Tool (z.B. Wordle) Wortwolken auf Russisch erstellen

- eine Webseite (z.B. mit Wordpress) erstellen und bearbeiten

- Links kürzen, um die Fehlereingabe zu minimieren

- sich mit Fragen des Datenschutzes im Internet auskennen

- Aufgaben zum Sprachenlernen mit Vorlagen/Modulbausteinen erstellen, z.B. auf https://learningapps.org oder mit SMART Lab /Activity Builder

- mit einer Dokumentenkamera arbeiten

- Standbilder zu Videos erstellen und Untertitel zu Videoausschnitten hinzufügen

die einen bestimmten Fachbegriff beinhalten, z.B. mit dem IWB erstellte Tafelbilder, mit einer Dokumentenkamera arbeiten, an einem Webinar teilnehmen, einen Tandembogen erstellen. Zu Semesterbeginn waren sechs Kompetenzformulierungen für gut ein Fünftel der Befragten nicht klar, wohingegen zum Ende des Semesters öfter gewusst wurde, was sich hinter den Termini verbirgt. Das am häufigsten nicht verstandene Item „sich in ein Doodle eintragen“ konnte jedoch von 17 Studierenden auch zum Semesterende nicht dechiffriert werden. 
- $\quad$ mit einem Tool wie Quizlet oder Goconqr virtuelle Lernkartensets erstellen

- Präsentationen für die Arbeit mit der interaktiven Tafel (z.B. mit SMART Notebook, ActivInspire) erstellen

- Schüler für die Seriosität von Internetquellen sensibilisieren können

- einen Unterrichtsmanager zu einem aktuellen Lehrwerk (z.B. Диалог) benutzen

- $\quad$ eine Online-Umfrage erstellen

- mit einem entsprechenden Tool Kreuzworträtsel in russischer Sprache erstellen

- Potenziale und Grenzen von programmiertem Feedback zu OnlineÜbungen und -Tests einschätzen

- eine interaktive Tafel (interaktives Whiteboard, SMART-Board, ActivBoard) bedienen

- ein Online-Portfolio bearbeiten

- ein Webinar für Andere anbieten.

Die Kompetenzen auf der Wunschliste zu Studierenden stimmen weitgehend mit den Kompetenzen überein, über die die Befragten bis dato nicht verfügen (vgl. Tab. 5). Die zusätzlichen Anmerkungen einiger Studierender in den Fragebögen geben weiteren Aufschluss zu Bereichen, in denen die Studierenden ihre Kompetenzen als ausbaufähig einschätzen. So wurde das Item 1.16 „Ich kann für das Sprachenlernen hilfreiche Programme/Apps auf meinen Computer herunterladen" u.a. wie folgt kommentiert:

- „Ich kann sie theoretisch herunterladen, weiß aber nicht, wo ich sie finden kann."

- „Hierzu würde mich interessieren, welche hilfreichen Programme es gibt."

- „Ich würde gern wissen, welche Programme/Apps zu empfehlen sind." 
Auch bestimmte Strategien der Lehramtsstudierenden werden aus den Anmerkungen deutlich, z.B. „Kenne ich gewisse Funktionen eines Programms nicht, dann schaue ich auf youtube.com oder google.de nach Videos, die diese Inhalte erläutern. Nach ungefähr 20 Minuten müsste ich die Betonungszeichen einfügen können." Zum Teil erfahren wir durch die Kommentare auch, welche Items falsch bzw. nicht verstanden wurden, bspw. „Falls ein Skype-Unterricht auch als 'Webinar' bezeichnet werden kann, habe ich es dann verwendet“ oder „Programmiertes Feedback? Was bedeutet das?“.

Um die subjektiven Theorien zur Nützlichkeit der Medien im Russischunterricht zu ermitteln, wurde auch nach Kompetenzen gefragt, die die Studierenden als irrelevant für ihren späteren Lehrerberuf ansehen (FF 5). Dies betraf aus Sicht der Studierenden die folgenden Aspekte:

- einen Blog (auf Russisch) erstellen

- sich an einem russischsprachigen Forum beteiligen

- Teil eines sozialen Netzwerks sein, in dem Russisch verwendet wird

- interessante Blogs kommentieren

- wissen, wie soziale Netzwerke (z.B. Facebook, Twitter, vKontakte, Odnoklassniki, Instagram, Snapchat) funktionieren

- Videos auf YouTube hochladen

- Graphiken in Excel erstellen und bearbeiten

- Internettelefon (z.B. Skype) bewusst zum Sprachenlernen (Hörverstehen, Sprechen), z.B. mit einem Tandempartner, nutzen

- eine Webseite (z.B. mit Wordpress) erstellen und bearbeiten

- QR-Codes scannen, erstellen und ihre Funktion kennen

- wissen, wie ich zu viel Werbung im Internet umgehen kann

- ein Webinar für Andere anbieten

- Kommentare anderer Internetnutzer (z.B. auf Facebook, YouTube usw.) entsprechend einordnen

- eine Videokonferenz organisieren und moderieren. 
Es muss angemerkt werden, dass diese Kompetenzen maximal von $23 \%$ der Studierenden als irrelevant eingeschätzt wurden. Die Anzahl der als unwichtig angesehenen Kompetenzen hat sich allerdings zum Ende des Semesters von drei (oben fett markiert) auf 14 erhöht.

Auch an den Reflexionen der Studierenden in ihren Portfolios lassen sich Einstellungen im Bereich Medienkompetenz ablesen. So schreibt eine Studentin: ,Was meine Medienkompetenz angeht, finde ich, dass ich kein Anfänger am PC, Handy, IWB, OHP und im Internet bin, jedoch kommt immer Neues dazu, was man lernen sollte. Vor allem was rechtliche Grundlagen oder das Prüfen von Quellen und Informationen angeht, habe ich noch viel zu lernen. Darin sollte man als Lehrkraft auch ein fundiertes Wissen aufweisen."

Ihre Kommilitonin sieht das ähnlich: „Что касается меня как учителя русского языка, то я чувствую себя на уроке технически компетентной. В силу того, что информационные технологии и гаджеты играют большую роль и в моей жизни, проблем с использованием таких средств обучения, как телевизоры, проигрыватели, компьютеры, смартфоны и социальные сети, в большинстве своем у меня не возникает. [...] И все же, есть аспекты, которые были мне незнакомы до начала семестра. Например, только на практике мне удалось раскрыть для себя возможности интерактивной доски и поработать с программой Unterrichtsmanager, которая заметно облегчает объяснение материала, постановку различных задач и обеспечивает наглядность. Также я узнала о таких платформах, как Moodle, и получила больше знаний в таких областях, как работа с Word документом, внесение в него исправлений и специальных знаков. Помимо этого, я узнала о существовании многих полезных сайтов, таких как, например, learningapps.org или udarenieru.ru. Следует сказать, что некоторые юридические вопросы об использовании информации, лицензии и защите прав правообладателя остались мне до конца неясны. Мне кажется, что в 
этой области мне необходимы знания специалиста, и я надеюсь, что в будущем смогу больше узнать о данных темах“.

Eine andere Studentin schreibt: „Im Laufe des letzten Semesters war es definitiv mein Ziel meine Medienkenntnisse zu erweitern. Ich wollte vor allem den Umgang mit Word und PowerPoint erweitern und dies ist mir zum Teil gelungen. Jedoch möchte ich in Zukunft öfter Angebote an der Universität wahrnehmen, die das Üben oder Ausprobieren mit neuen Medien anbieten, da ich bisher davor zurückgeschreckt bin. Im Laufe des letzten halben Jahres ist mir jedoch bewusst geworden, dass der Umgang und die Nutzung von verschiedenen Medien und Medienkompetenz im Lehrerberuf unumgänglich ist und sogar einen großen positiven Beitrag leisten kann.“

Eine weitere Studentin reflektiert, dass Kinder und Jugendliche zwar oft über technische Medienkompetenzen verfügen, im Bereich der kritischen Medienkompetenz jedoch durchaus Lernbedarf haben, auf den sie als künftige Russischlehrerin eingehen will: „Как правило, школьники уже обладают необходимой для урока русского языка медиакомпетентностью, что выражается в их умении обращаться с помощью современных технологий. Тем не менее, их медиакомпетентность является неполной, так как обычно они не обладают способностью к критической оценке используемых средств. Например, большое количество учеников пользуется для перевода незнакомых слов сервисом google translate, который не указывает все значения слова и поэтому зачастую приводит к механическому или ошибочному переводу.“

Anastasia Drackert und Katrin Bente Karl (Hg.), Didaktik der slawischen Sprachen

Beiträge zum 2. Arbeitskreis in Innsbruck (19.02.-20.02.2018)

(C) 2019 innsbruck university press, ISBN 978-3-903187-80-1, DOI 10.15203/3187-80-1 


\section{Diskussion und Ausblick}

Erfolgreiche Lehrpersonen zeichnen sich nicht nur durch Kompetenzen aus, sondern auch durch Einstellungen (vgl. Zierer 2018). Während die meisten Kommentare zum Fragebogen und die schriftlichen Reflexionen der Studierenden in ihren Lehramtsportfolios für eine generell aufgeschlossene Grundhaltung gegenüber Neuem im Bereich Medienkompetenz sprechen, besteht bei einigen Überzeugungen, z.B. hinsichtlich der Irrelevanz bestimmter Kompetenzen für den schulischen Russischunterricht, noch Handlungsbedarf. So genügt die Kompetenz der Nutzung sozialer Netzwerke als Präsentations-, Informations- und Kommunikationsmedien nicht. „Hinzukommen muss das Wissen bzw. Bewusstsein über Wert und Notwendigkeit der Privatheit von Daten und der Respekt vor der Unantastbarkeit der digitalen Identität anderer Menschen" (GFD 2018: 3). Ebenso ist es im Sinne der kritischen Medienkompetenz notwendig, dass Fremdsprachenlehrende Kommentare anderer Internetnutzer auf diversen Plattformen und in sozialen Medien in Bezug auf ihre Relevanz, Aussagekraft und ihren Wahrheitsgehalt einschätzen können. Wenn die Lernenden sich im russischen Internet bewegen, sollten sie wissen, wie sie Werbung umgehen können, und die Russischlehrkraft sollte ihnen dieses Wissen vermitteln können.

Bei den von uns Befragten handelt es sich um eine recht heterogene Gruppe Lehramtsstudierender aus verschiedenen Semestern und Schulformen, die sich sowohl in ihren Sprachlernbiografien (Fremdsprachenlernende, HerkunftssprecherInnen, MuttersprachlerInnen) als auch in ihren Medienkompetenzen unterscheiden. Dennoch geben die Ergebnisse aus der Befragung erste Antworten auf die eingangs formulierten Forschungsfragen. Sicher ist bei einer solchen Art der Befragung eine gewisse Tendenz der sozialen Erwünschtheit im Antwortverhalten nie ganz auszuschließen. Dennoch werden v.a. die Bereiche, in denen die Studierenden 
noch Unsicherheiten aufweisen und sich eine Verbesserung ihrer Kompetenzen wünschen, deutlich. Die Kombination mit qualitativen Daten (Kommentare zu den Fragebogen-Items, Reflexion der eigenen Medienkompetenz im Portfolio) gestattet zusätzliche Einblicke in die subjektiven Überzeugungen der Studierenden.

Für eine Weiterarbeit mit dem Fragebogen in den nächsten Semestern erscheint es sinnvoll, die große Anzahl der Items zu reduzieren und bspw. diejenigen auszuschließen, in denen mehr als 90\% der Studierenden über sehr gute Kompetenzen verfügen. Eine Triangulation mit qualitativen Interviews, aber auch mit Ergebnissen aus Gruppendiskussionen im Seminar würde es erlauben, noch mehr in die Tiefe zu gehen, z.B. um mehr darüber zu erfahren, wie Medienkompetenz aus Sicht der Studierenden erworben wird, weshalb bestimmte Kompetenzen von ihnen als irrelevant angesehen werden und was die Studierenden unternehmen wollen, um sich die aus ihrer Sicht erstrebenswerten Kompetenzen anzueignen. Wünschenswert wäre zudem die Weiterführung der Longitudinalstudie über einen längeren Studienabschnitt sowie eine Ausweitung auf weitere Fremdsprachen bzw. Lehramtsfächer.

Die Liste der Studierenden in Bezug auf wünschenswerte Medienkompetenzen kann ein Ausgangspunkt für die systematische Medienkompetenzvermittlung in der Russischlehrkräfteausbildung sein. So haben an der Universität Leipzig im Modul „Didaktik der slawischen Sprachen 3“ alle Studierenden im Sinne der alternativen Methode Lernen durch Lehren (LdL) im Laufe des folgenden Semesters in einer kleinen Mini-Präsentation ihren KommilitonInnen ein Webtool bzw. eine bestimmte Medienteilkompetenz vorgestellt, das/die sie sich selbst vor kurzem angeeignet haben, bspw. die Ergänzung eines Wikipedia-Eintrags mit Bezug zur russischen Sprache, die Erstellung eines Lexikoneintrags einer bisher noch fehlenden Vokabel in einem Online-Wörterbuch, das Hinzufügen von Untertiteln $\mathrm{zu}$ einem russischen Trickfilm, die Erstellung eines Lernvideos für den Russischunterricht oder eines Suchrätsels mithilfe der Webseite 
Головоломка. Die Studierenden erstellten zudem im Laufe des Semesters selbst eine Übung auf https://learningapps.org/ für den Russischunterricht, die sie didaktisch-methodisch begründet in eine längere Übungssequenz zu einem bestimmten grammatischen oder orthografischen Phänomen einbinden mussten. Schließlich wurde ein Webinar zu virtuellen Helfern für den Russischunterricht durchgeführt, an dem Lehramtsstudierende aller drei Ausbildungsstandorte gemeinsam teilnahmen.

Neben den Kompetenzen, über die die Befragten noch gar nicht verfügen, sollten insbesondere diejenigen in den Didaktikveranstaltungen thematisiert werden, die von einigen Studierenden als irrelevant eingestuft wurden. So sehen sich russische MuttersprachlerInnen bzw. HerkunftssprecherInnen meist nicht (mehr) als Sprachenlernende des Russischen und bewerten damit zusammenhängende mediale Sprachlernaktiväten wie die Nutzung von Skype zur Verbesserung mündlicher Fertigkeiten als irrelevant. Für Fremdsprachenlernende haben Skype-Sitzungen mit Muttersprachlern jedoch als Quelle für reichen authentischen Input sowie als Medium einer authentischen Interaktion großes Lernpotenzial. Lehramtsstudierende, die selbst HerkunftssprecherInnen sind, sollten Interesse an medialen Gelegenheiten haben, die ihnen den Ausbau ihrer schriftsprachlichen Kompetenzen ermöglichen.

Schließlich wurden die Autorinnen dieses Beitrags durch die Ergebnisse dieser Erhebung stärker für die Lernbedürfnisse ihrer Studierenden im Bereich Medienkompetenz sensibilisiert. Ein einziges Seminar allein kann diese Querschnittsaufgabe für die Lehrerausbildung jedoch nicht bewältigen; Medienkompetenzen müssten in allen Veranstaltungen der Russischdidaktik, der Sprachpraxis und der Fachwissenschaften mitgedacht und weiterentwickelt werden.

Die Autorinnen haben zudem durch die intensive Beschäftigung mit dem Thema in den vergangenen Monaten selbst ihre Medienkompetenzen ausbauen können. Hochschullehrende, die sich selbst als Lernende sehen und 
den Studierenden zeigen, dass sie auch von ihnen lernen wollen, können sicher zu einer größeren Aufgeschlossenheit in Bezug auf neue Medienanforderungen bei ihren Studierenden und künftigen Fremdsprachenlehrenden beitragen (vgl. Burwitz-Melzer et al. 2019).

\section{Literatur}

Adler, I., Boiselle, T., Breitsprecher, R., Chwoika, A., Heller, M., Müller, J., Nadchuk, E., Seidel, A., Semashkina, L. \& Steinbach, A. (2016). Dialog 1. Lehrwerk für den Russischunterricht. Unterrichtsmanager. Berlin: Cornelsen.

Baake, D. (1997). Medienpädagogik. Tübingen: Niemeyer.

Beatty, K. (2010). Teaching and researching Computer Assisted Language Learning. London: Pearson.

Blömeke, S. (2017). Erwerb medienpädagogischer Kompetenz in der Lehrerausbildung. Modell der Zielqualifikation, Lernvoraussetzungen der Studierenden und Folgerungen für Struktur und Inhalte des medienpädagogischen Lehramtsstudiums. MedienPädagogik, Medien-Päd.Retro: Jahrbuch Medienpädagogik 3 (2003), 231-244. doi:10.21240/mpaed/retro/2017.07.13.X.

Burwitz-Melzer, E., Riemer, C. \& Schmelter, L. (Hrsg.) (2019). Digitaler Wandel. Arbeitspapiere der 39. Frühjahrskonferenz zur Erforschung des Fremdsprachenunterrichts. (= Giessener Beiträge zur Fremdsprachendidaktik). Tübingen: Narr Francke Attempto.

Дженкова, Е.А. \& Мозгалина, А.И. (2010). Е-Тандем как вид дистанционного обучения. В: Слышкин, Г.Г. \& Бессарабова, И.С. (ред.), Меняющая коммуникачия в меняющемся мире - 5 . Сборник статей. Волгоград, 73-75.

Europäische Union (2017). Digitale Kompetenz Lehrender. Verfügbar unter: 
https://ec.europa.eu/jrc/sites/jrcsh/files/digcompedu_leaflet_de_201 8-01.pdf [31.10.2018].

Europarat (Hrsg.) (2001). Gemeinsamer europäischer Referenzrahmen für Sprachen. Lehren, lernen, beurteilen. Berlin: Langenscheidt.

Ferrari, A. (2013). DigComp: A framework for Developing and Understanding Digital Competence in Europe, Seville: JRC-IPTS. Verfügbar unter:

http://ipts.jrc.ec.europa.eu/publications/pub.cfm?id=6359

[31.10.2018].

GFD - Gesellschaft für Fachdidaktik e.V. (2018). Fachliche Bildung in der digitalen Welt. Positionspapier der Gesellschaft für Fachdidaktik. Verfügbar unter:

http://www.fachdidaktik.org/veroeffentlichungen/positionspapiereder-gfd/ [27.10.2018].

Grünewald, A. (2017). Medienkompetenz. In C. Surkamp (Hrsg.), Metzler Lexikon Fremdsprachendidaktik. Ansätze - Methoden - Grundbegriffe (245-246). Stuttgart: J.B. Metzler.

Guhl, M. (2008). RuSkeLL: Ein Internetkorpus für Russischlehrer und Russischlerner. Praxis Fremdsprachenunterricht Russisch 4/2018, 12.

Heim, K. \& Ritter, M. (eds.) (2012). Computer-Assisted Language Learning. Paderborn: Schöningh.

KMK (2016). Bildung in der digitalen Welt. Strategie der Kultusministerkonferenz Verfügbar unter:

https://www.kmk.org/fileadmin/Dateien/pdf/PresseUndAktuelles/2016/Bildung_digitale_Welt_Webversion.pdf [31.10.2018].

KMK (2017). Ländergemeinsame inhaltliche Anforderungen für die Fachwissenschaften und Fachdidaktiken in der Lehrerbildung (Beschluss der Kultusministerkonferenz vom 16.10.2008 i.d.R. vom 12.10.2017). Bonn.

Middendorff, E. (2002). Computernutzung und Neue Medien im Studium. Ergebnisse der 16. Sozialerhebung des Deutschen Studentenwerkes

Anastasia Drackert und Katrin Bente Karl (Hg.), Didaktik der slawischen Sprachen

Beiträge zum 2. Arbeitskreis in Innsbruck (19.02.-20.02.2018)

(C) 2019 innsbruck university press, ISBN 978-3-903187-80-1, DOI 10.15203/3187-80-1 
(DSW) durchgeführt von HIS Hochschul-Informations-System. Bonn: BMBF.

MSW - Ministerium für Schule und Weiterbildung des Landes NordrheinWestfalen (2014). Kernlehrplan Gymnasium/Gesamtschule in Nordrhein-Westfalen. Verfügbar unter: https://www.schulentwicklung.nrw.de/lehrplaene/lehrplan/98/ KLP_GOSt_Russisch.pdf [31.10.2018].

Newby, D., Allan, R., Fenner, A.-B., Jones, B., Komorowska, H. \& Soghikyan, K. (2007). European Portfolio for Student Teachers of Languages. A reflection tool for language teacher education.

Council of Europe. Verfügbar unter: archive.ecml.at/mtp2/fte/pdf/c3_epostl_e.pdf [27.10.2018]. Rösler, D. (2010). E-Learning Fremdsprachen - eine kritische Einführung. Tübingen: Stauffenburg.

SenBJF \& MBJS - Berliner Senatsverwaltung für Bildung, Jugend und Familie \& Ministerium für Bildung, Jugend und Sport des Landes Brandenburg (2015). Rahmenlehrplan für die Jahrgangsstufen 1-10 der Berliner und Brandenburger Schulen. Teil B. Fachübergreifende Kompetenzentwicklung. Verfügbar unter: https://bildungsserver.berlinbrandenburg.de/fileadmin/bbb/unterricht/rahmenlehrplaene/Rahme nlehrplanprojekt/amtliche_Fassung/Teil_B_2015_11_10_WEB.pdf [28.10.2018].

SMK - Sächsisches Staatsministerium für Kultus (2011). Lehrplan Russisch Gymnasium. Dresden.

SMWK - Staatsministerium für Wissenschaft und Kunst (2018). Strategie zur Digitalisierung in der Hochschulbildung. Verfügbar unter: studieren.sachsen.de/download/Strategiepapier_Digitalisierung.pdf [27.10.2018].

Wapenhans, H. (2014). Medieneinsatz im Russischunterricht. In A. Bergmann (Hrsg.), Fachdidaktik Russisch. Eine Einführung (253-265). Tübingen: Narr. 
Zierer, K. (2018). Lernen 4.0 - Pädagogik vor Technik. Möglichkeiten und Grenzen einer Digitalisierung im Bildungsbereich. (2. Aufl.) Baltmannsweiler: Schneider. 